\title{
Profilaxia pré-exposição ao HIV e territorialidade nas publicações científicas brasileiras
}

\author{
HIV pre-exposure prophylaxis and territoriality in Brazilian scientific publications
}

Profilaxis previa a la exposición al VIH y territorialidad en las publicaciones científicas brasileñas

Mayra Savi Gonçalves ${ }^{1}$, Florêncio Mariano da Costa-Júnior ${ }^{1}$.

\begin{abstract}
RESUMO
Objetivo: Mapear estudos recentes que abordam fatores territoriais que podem contribuir para as estratégias de Profilaxia Pré-Exposição (PrEP) ao HIV. Métodos: Trata-se de uma revisão integrativa, na qual para coletar os dados se utilizou os descritores "PrEP" e "Profilaxias HIV". A base de dados selecionada foi o portal regional da Biblioteca Virtual em Saúde (BVS). Como critérios de inclusão, foram considerados estudos em língua portuguesa, estudos publicados no período de 2010 a 2020 e estudos empíricos produzidos no Brasil. Resultados: Os achados sinalizam para a escassez de estudos atuais sobre os métodos de profilaxia ao HIV que discutam os aspectos particulares de contextos regionais do território brasileiro, e os existentes excluem, quase completamente, a população residente no interior dos estados. Considerações finais: Torna-se urgente a realização de pesquisas empíricas com populações brasileiras não metropolitanas, estudos que discorram sobre o HIV, seus métodos de prevenção, comportamentos de risco, acesso e uso dos programas de PrEP. Visando garantir os princípios fundamentais do SUS, as profilaxias precisam se articular com base nas particularidades das regiões não metropolitanas e em suas dimensões socioculturais.
\end{abstract}

Palavras-chave: Profilaxia pré-exposição, Território sociocultural, HIV.

\begin{abstract}
Objective: To map recent studies that address territorial factors that may contribute to HIV pre-exposure prophylaxis (PrEP) strategies. Methods: This is an integrative review, in which the descriptors "PrEP" and "HIV Prophylaxis" were used to collect data. A selected database was the regional portal of the Virtual Health Library (VHL). As inclusion of inclusion, studies in Portuguese were considered, studies published in the period from 2010 to 202020 and empirical studies in Brazil. Results: Findings point to the resident methods, particular to existing and detailed studies on the regional aspects of the Brazilian territory, almost completely the population in the interior of the states. Final considerations: It is urgent to carry out empirical research with non-metropolitan Brazilian populations, studies that discuss HIV, its prevention methods, methods of methods, access and use of PrEP programs. In order to guarantee the fundamental principles of the SUS, such as prophylaxis, they need to be articulated based on the particularities of non-metropolitan regions and their sociocultural dimensions.
\end{abstract}

Keywords: Pre-exposure prophylaxis, Sociocultural territory, HIV.

\section{RESUMEN}

Objetivo: mapear estudios recientes que abordan factores territoriales que pueden contribuir a las estrategias de profilaxis previa a la exposición (PrEP) al VIH. Métodos: Esta es una revisión integradora, en la que se utilizaron los descriptores "PrEP" y "Profilaxis del VIH" para recopilar datos. Una base de datos seleccionada fue el portal regional de la Biblioteca Virtual en Salud (BVS). Como inclusión de inclusión, se consideraron estudios en portugués, estudios publicados en el período de 2010 a 202020 y estudios empíricos en Brasil. Resultados: Los hallazgos apuntan a los métodos residentes, particulares a los estudios existentes y

1 Universidade Estadual Paulista (UNESP), Araraquara - SP. *E-mail: savi.mayra@gmail.com 
detallados sobre aspectos regionales del territorio brasileño, casi en su totalidad la población del interior de los estados. Consideraciones finales: Es urgente realizar investigaciones empíricas con poblaciones brasileñas no metropolitanas, estudios que discutan el $\mathrm{VIH}$, sus métodos de prevención, métodos de métodos, acceso y uso de los programas de PrEP. Para garantizar los principios fundamentales del SUS, como la profilaxis, es necesario articularlos a partir de las particularidades de las regiones no metropolitanas y sus dimensiones socioculturales.

Palabras clave: Profilaxis pre-exposición, Territorio sociocultural, VIH.

\section{INTRODUÇÃO}

Segundo o Boletim Epidemiológico do Vírus da Imunodeficiência Humana e Síndrome da Imunodeficiência Adquirida (HIV/aids), divulgado em dezembro de 2020 pela Secretaria de Vigilância em Saúde e Ministério da Saúde, de 1980 a junho de 2020, foram identificados 1.011.617 casos de infecção pelo HIV no Brasil. Entre indivíduos contaminados maiores de 13 anos, em 2019, a principal via de transmissão foi a sexual, tanto em homens $(79,3 \%)$ quanto em mulheres (87,3\%) (MINISTÉRIO DA SAÚDE, 2020).

Os homens jovens ainda são a parte da população mais impactada pelo vírus HIV. Em 2019, a maior taxa de detecção foi de 52 casos a cada 100 mil habitantes, e ocorreram entre os indivíduos na faixa etária de 25 a 29 anos, superando as taxas de detecção em homens de 30 a 34 anos e de 35 a 39 anos, que eram mais prevalentes até o ano de 2015. No Brasil, entre os homens acometidos pelo HIV, observou-se o predomínio da categoria de exposição homo/bissexual (39,8\%) (MINISTÉRIO DA SAÚDE, 2020).

Mesmo sendo os mais impactados pelo vírus, a população masculina encontra barreiras para conhecer seu status sorológico. Os serviços de saúde que atendem essa população não oferecem os testes cotidianamente, diminuindo assim a probabilidade dos homens o realizarem. Alguns aspectos normativos da masculinidade também influenciam o não acesso à testagem (COSTA-JÚNIOR FM, 2014; KNAUTH DR, et al., 2020 e PARKER RG, 2000).

No que se refere à orientação sexual, de acordo com o Ministério da Saúde (2020), a diferença na incidência de novos casos entre as categorias homo/bissexual e heterossexual é de menos de um por cento. Quanto à diferença do percentual de infecção entre os sexos, no ano de 2019, novos casos prevaleceram entre os homens, sendo que a proporção foi de 26 homens para cada 10 mulheres. Entre os homens infectados pelo HIV, no ano de 2019, predomina a categoria de exposição homo/bissexual, nas regiões Sudeste e Centro-Oeste, enquanto nas demais regiões, o predomínio foi de exposição heterossexual.

Desde 1980, no início da epidemia, até 31 de dezembro de 2019, foram notificados no Brasil, 349.784 óbitos derivados do HIV. A região Sudeste concentra a maior parcela desses óbitos, totalizando $57,7 \%$ e a região Norte aparece em última posição, com 5,3\% do total de óbitos notificados (MINISTÉRIO DA SAÚDE, 2020).

Ao observarmos as diferenças dos índices em determinadas regiões, o território passa a ser uma categoria de análise social, pois se torna, cada vez mais, um elemento poderoso para a compreensão da dinâmica socioespacial, isto é, da sociedade e de seus territórios, sua história lá onde ela se faz. A autora corrobora ainda que o poder local vai se tornando, paulatinamente, mais importante do que o poder nacional. Este dependerá cada vez mais daquele (SOUZA MAA, 2004).

Segundo Santos MA (1999), o território consiste em um lugar composto por dimensões culturais e físicas e é, sobretudo, um espaço de relações de poder, de informações e de interações. Desse modo, a identificação e localização dos serviços, seus usos pela população e sua importância para os fluxos das pessoas e de insumos são de grande relevância para o conhecimento da dinâmica social, dos hábitos e costumes e para a determinação de vulnerabilidades de saúde, originadas nas interações de grupos humanos em determinados espaços territoriais (MONKEN M, et al., 2008).

Atualmente, os métodos preventivos do HIV são explanados em campanhas nacionais, estaduais e municipais. Entretanto, apesar das campanhas e da conscientização de sua eficácia, por exemplo, o preservativo masculino, conhecido popularmente como camisinha, não é unanimidade entre os usuários. A 
dificuldade de uso contínuo de camisinha é um dos indicativos que anuncia a necessidade da ampliação da oferta de novas tecnologias de prevenção visando à redução da incidência do HIV (MAKSUD I, et al., 2015).

A Profilaxia Pré-Exposição (PrEP) ao HIV norteia as novas discussões sobre os novos métodos de prevenção, trazendo novas possibilidades para o controle global da epidemia (ZUCCHI EM, et al., 2018). Em 1 de dezembro de 2017, no dia mundial da aids, a PrEP foi oficialmente lançada no Brasil. Seu lançamento seguiu os critérios de inclusão de participantes de acordo com o Protocolo Clínico e Diretrizes Terapêuticas para PrEP de Risco à infecção por HIV, emitido pelo Ministério da Saúde (LOPES JDS, et al., 2019).

A PrEP foi concebida na perspectiva da Prevenção Combinada (PC), uma estratégia global de prevenção ao HIV e às demais Infecções Sexualmente Transmissíveis (IST), cujo intuito é o uso simultâneo de abordagens biomédicas, comportamentais e programáticas, a fim de potencializar as respostas à epidemia de HIV/aids. Nesse sentido, constatou-se que abordagens prescritivas, baseadas no estímulo ao uso de um único método preventivo, como a camisinha, por exemplo, já não são mais suficientes como forma de prevenir a aids e as demais ISTs (POLIDORO M, et al., 2020).

Quando foi oficialmente implantada pelo Ministério da Saúde, a PrEP passou a ser ofertada exclusivamente no Sistema Único de Saúde (SUS). Inicialmente, algumas capitais brasileiras e alguns municípios médios foram contemplados com sua oferta. Quando incorporada ao SUS, alguns municípios já contavam com a disponibilização do método, por meio dos estudos demonstrativos anteriormente realizados (POLIDORO M, et al., 2020).

Com o intuito de contribuir para a discussão da implementação e da aceitabilidade da PrEP no Brasil e considerando a relevância em compreender as particularidades regionais na implementação de políticas de saúde, o presente estudo objetivou elaborar uma revisão integrativa de publicações brasileiras que trazem a lume análises acerca dos fatores territoriais que podem contribuir para as estratégias da Profilaxia PréExposição ao HIV.

\section{MÉTODOS}

O presente estudo realizou uma revisão integrativa sobre a PrEP, visando encontrar pesquisas empíricas com populações não metropolitanas, a fim de mapear estudos recentes que abordem esse método de prevenção em contextos regionais.

A busca foi realizada com os descritores "PREP OR Profilaxias HIV", e a base de dados selecionada para a realização da busca foi o portal regional da Biblioteca Virtual em Saúde (BVS). A escolha pela BVS se deu levando em consideração o fato de a plataforma contar com artigos científicos na área da saúde, na íntegra e de forma gratuita, e compilar as seguintes bases de dados: Literatura Latino-Americana e do Caribe em Ciências da Saúde (LILACS), Scientific Eletronic Library Online (ScIELO) e Literatura Internacional em Ciências da Saúde (MEDLINE).

Para a elegibilidade dos estudos que compõem essa revisão, foram considerados os seguintes critérios: 1) Estudos publicados em língua portuguesa; 2) Estudos publicados no período de 2010 a 2020: O período foi escolhido pois corresponde à validade de estudos dessa natureza; 3) Estudos empíricos produzidos no Brasil. A busca foi realizada em abril de 2021 e resultou, inicialmente, em 6986 estudos. A partir desses resultados, os critérios de inclusão foram aplicados, e 57 estudos atenderam aos critérios de inclusão, ou seja, menos de $1 \%$ do total de publicações encontradas levando-se em conta apenas os descritivos iniciais "PrEP OR Profilaxias HIV".

O passo seguinte consistiu na leitura dos títulos e resumos da seleção inicial de 57 estudos, com a finalidade de preencher o último critério de inclusão proposto: estudos empíricos produzidos no Brasil. Foram excluídos todos os artigos que utilizavam a sigla "prep" para outras denominações que não a profilaxia préexposição ao HIV.

Por fim, teses e dissertações cujo acesso ao texto completo não estava disponível e entrevistas relacionadas ao tema foram igualmente descartadas. 


\section{RESULTADOS E DISCUSSÃO}

Se considerarmos que, aplicando, inicialmente, apenas os descritivos "PREP OR Profilaxias HIV", o primeiro resultado obtido é de 6.986 estudos, e que, ao final da pré-seleção, esse número se reduz a 9, já nos deixa a hipótese que a temática em questão é pouco estudada e divulgada no Brasil e que pesquisas relacionadas a ela são escassas.

Sobre os resultados obtidos, o volume de publicações sobre a PrEP aparece em maior quantidade a partir de 2018, e a maior concentração dessas publicações encontra-se em periódicos de Enfermagem e de Saúde.

Para discorrermos melhor sobre os resultados, serão descartados os estudos sobre a PrEP em uma perspectiva generalista, ou seja, estudos que abordam a temática em outras perspectivas ou em outros países. Foram considerados estudos que abordam a temática e apresentam pesquisas realizadas no Brasil, ou análise histórica/documental acerca do HIV/aids ou da PrEP no Brasil.

A partir da triagem final realizada, efetuando as exclusões citadas anteriormente e desconsiderando estudos que apareceram em duplicidade, obtivemos um total de 15 estudos aptos para leitura completa, com o intuito de identificar e preencher o último critério de inclusão: pesquisas empíricas realizadas no Brasil. Dentre todos os estudos analisados, apenas nove constituíram a presente revisão integrativa (Figura 1).

Figura 1 - Processo de seleção amostral nas bases de dados utilizadas.

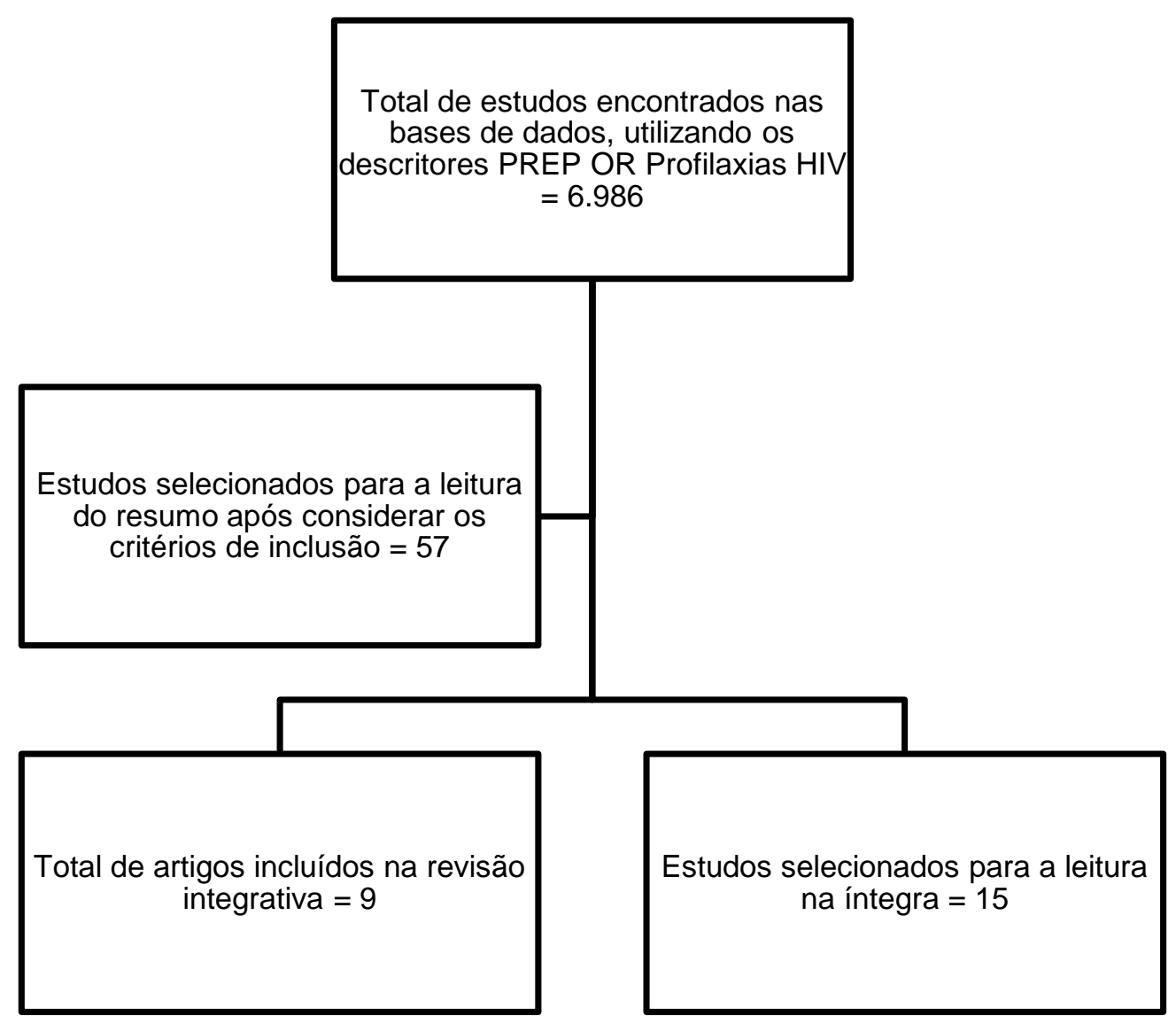

Fonte: Gonçalves MS e Costa-Júnior FM, 2022.

\section{Estudos sobre PrEP e contextos territoriais do Brasil}

Abaixo apresentaremos os estudos que abordam a PrEP em uma perspectiva que considera os territórios brasileiros, sua cultura e suas especificidades (Quadro 1). 
Quadro 1 - Estudos sobre PrEP e contextos territoriais do Brasil.

\begin{tabular}{|c|c|c|c|}
\hline Título & Autor e ano & TE & Objetivo \\
\hline $\begin{array}{l}\text { Conhecimento sobre HIV/Aids } \\
\text { implicações no estabelecimento de } \\
\text { parcerias entre usuários do Hornet. }\end{array}$ & $\begin{array}{l}\text { QUEIROZ AAFLN, et } \\
\text { al. (2018). }\end{array}$ & Estudo descritivo. & $\begin{array}{l}\text { Compreender o conhecimento de } \mathrm{HSH} \text {, a } \\
\text { respeito do HIV, nos relatos de homens que } \\
\text { utilizam o aplicativo de encontros Hornet. }\end{array}$ \\
\hline $\begin{array}{l}\text { Percepções sobre o uso da profilaxia pré- } \\
\text { exposição ao HIV (PrEP) e possível } \\
\text { compensação de risco entre homens que } \\
\text { fazem sexo com homens (HSH) e mulheres } \\
\text { transexuais (TRANS) potencialmente } \\
\text { elegíveis para o uso de PrEP no estudo } \\
\text { PrEP Brasil. }\end{array}$ & VILLELA LM (2018). & Estudo qualitativo. & $\begin{array}{l}\text { Verificar a existência de compensação de risco } \\
\text { entre potenciais usuários de PrEP, no estudo } \\
\text { PrEP Brasil. }\end{array}$ \\
\hline $\begin{array}{l}\text { Da evidência à ação: desafios do Sistema } \\
\text { Único de Saúde para ofertar a profilaxia pré- } \\
\text { exposição sexual (PrEP) ao HIV às pessoas } \\
\text { em maior vulnerabilidade. }\end{array}$ & $\begin{array}{c}\text { ZUCHI EM, et al. } \\
(2018) .\end{array}$ & Ensaio Clínico. & $\begin{array}{l}\text { Avaliar o perfil dos usuários da PrEP, suas } \\
\text { vantagens se a outros métodos preventivos e os } \\
\text { desafios para o uso contínuo, protetivo e seguro, } \\
\text { a partir de resultados obtidos em estudos } \\
\text { brasileiros e internacionais. }\end{array}$ \\
\hline $\begin{array}{l}\text { Diversidade e liberdade sexual: Defensoria } \\
\text { Pública, movimentos sociais e a PrEP no } \\
\text { SUS. }\end{array}$ & $\begin{array}{l}\text { SILVA RATML } \\
\text { (2018). }\end{array}$ & Análise Qualitativa. & $\begin{array}{l}\text { Analisar a necessidade da contribuição de } \\
\text { movimentos sociais e da Defensoria Pública do } \\
\text { estado de São Paulo no processo de } \\
\text { incorporação da PrEP ao SUS. }\end{array}$ \\
\hline $\begin{array}{l}\text { Risco, biomedicalização e aids: cobertura } \\
\text { jornalística sobre métodos biomédicos de } \\
\text { prevenção ao HIV. }\end{array}$ & COSTA SLM (2019). & Estudo qualitativo. & $\begin{array}{l}\text { Analisar a cobertura jornalística sobre os } \\
\text { métodos preventivos ao HIV: PrEP, a PEP e o } \\
\text { TcP, desde as primeiras notícias a respeito do } \\
\text { tema, até o fim de } 2017 \text {. }\end{array}$ \\
\hline $\begin{array}{l}\text { Do AZT à PrEP e à PEP: aids, HIV, } \\
\text { movimento LGBT e jornalismo. }\end{array}$ & $\begin{array}{l}\text { CARVALHO CA, } \\
\text { AZEVÊDO JHPC } \\
\text { (2019). }\end{array}$ & Revisão de literatura. & $\begin{array}{l}\text { Verificar as coberturas jornalísticas sobre } \\
\text { movimentos LGBTQIA+, sobre a homofobia, o } \\
\text { HIV e a AIDS. }\end{array}$ \\
\hline $\begin{array}{l}\text { Aids em cartazes: representações sobre } \\
\text { sexualidade e prevenção da aids nas } \\
\text { campanhas de 1. de Dezembro no Brasil } \\
(2013-2017) \text {. }\end{array}$ & $\begin{array}{l}\text { LERMEN HS, et al. } \\
(2019) .\end{array}$ & $\begin{array}{l}\text { Análise de conteúdo } \\
\text { descritivo e } \\
\text { documental. }\end{array}$ & $\begin{array}{l}\text { Identificar as representações da sexualidade e os } \\
\text { métodos de prevenção apresentadas nas } \\
\text { campanhas do dia mundial da luta contra a aids, } \\
\text { entre os anos de } 2013 \text { e } 2017 \text {. }\end{array}$ \\
\hline $\begin{array}{l}\text { Responsabilidade, consentimento e } \\
\text { cuidado. Ética e moral nos limites da } \\
\text { sexualidade. }\end{array}$ & $\begin{array}{l}\text { BARRETO VHS } \\
(2020) .\end{array}$ & Estudo observacional. & $\begin{array}{l}\text { Identificar e discutir a respeito dos termos } \\
\text { "responsabilidade", "consentimento" e "cuidado" } \\
\text { em grupos de pessoas adeptas à práticas } \\
\text { sexuais classificadas como "de risco". }\end{array}$ \\
\hline $\begin{array}{l}\text { O reaparecimento da homossexualidade } \\
\text { masculina nas estratégias de prevenção da } \\
\text { infecção por HIV: reflexões sobre a } \\
\text { implementação da PrEP no Brasil. }\end{array}$ & $\begin{array}{l}\text { BARP LFG, } \\
\text { MITJAVILA MR } \\
\text { (2020). }\end{array}$ & Analise Qualitativa. & $\begin{array}{l}\text { utilizam-se do texto "O desaparecimento da } \\
\text { homossexualidade" de Nestor Perlongher para } \\
\text { estabelecer uma relação entre a PrEP e o } \\
\text { "reaparecimento" da homossexualidade no } \\
\text { Brasil. }\end{array}$ \\
\hline
\end{tabular}

\section{Conclusão}

Os usuários do Hornet possuem conhecimento insuficiente sobre medidas de prevenção do HIV/aids, principalmente quando se descarta o uso do preservativo masculino.

Os participantes do estudo PrEP Brasil percebem seu alto risco de infecção pelo HIV. Existe a necessidade de vigilância epidemiológica para monitorar a compensação de risco nos contextos de assistência à saúde.

Foram identificadas disparidades entre o que é orientado pelas diretrizes do Ministério da Saúde para implementação da PrEP e o que de fato foi encontrado na estrutura dos serviços públicos que permitem o acesso à profilaxia.

Defender a PrEP é defender o direito à liberdade sexual ressignifica e, de certa forma, refunda os movimentos sociais de defesa de direitos das pessoas que vivem com HIV/aids.

Há uma pouca quantidade de publicações jornalísticas acerca dos métodos preventivos de uma maneira informativa descobertas, as reportagens visam mais avanços científicos e a biomedicalização. Para alguns veículos midiáticos, a PrEP vem sendo exposta como um abandono do sexo seguro, por meio de descrições moralistas e estereotipadas.

Os aspectos relacionados ao uso do preservativo perderam a força nas campanhas, visto que a individualização do cuidado vem se acirrando sob a responsabilização do sujeito de realizar a testagem.

A PrEP é retratada no artigo integrando a perspectiva da responsabilidade e de autocuidado de seus usuários.

O estudo defende que a PrEP possibilita o retorno dos homossexuais aos consultórios médicos e, mais do que isso, opera como objeto discursivo de problematizações de caráter sanitário e social.

Fonte: Gonçalves MS e Costa-Júnior FMD, 2022. 
Queiroz AAFLN, et al. (2018), realizaram um estudo descritivo, considerando o relato de 30 usuários do aplicativo Hornet: homens, acima de 18 anos que residiam em Teresina/PI ou Ribeirão Preto/SP, que estavam online no aplicativo no momento da coleta de dados e ter ativado em seu perfil a opção "Saiba seu status" (SKY). Entre os participantes, prevaleceu a faixa etária adulto-jovens (71,3\%), com idade entre 18 e 25 anos, ensino médio completo $(52,3 \%)$ e sem renda própria $(67,5 \%)$.

De acordo com os dados apresentados pelo estudo, as relações estabelecidas pelo aplicativo podem deixar o usuário em uma situação vulnerável, através de comportamentos que têm o potencial de exposição ao risco de infecção pelo HIV. Porém, apesar dessa conclusão, a avaliação desse conhecimento foi extremamente limitada e restringiu-se ao preservativo masculino (QUEIROZ AAFLN, et al., 2018).

Villela LM (2018) discorre a respeito da compensação de risco e de atitudes e crenças entre potenciais usuários de PrEP, apresentando uma análise secundária do estudo PrEP Brasil. No cenário da prevenção ao HIV, a compensação de risco seria uma modificação no comportamento dos usuários de PrEP afim de reduzirem o uso da camisinha, aumentarem o comportamento de risco sexual e assim reduzirem o benefício do uso da profilaxia, devido ao aumento de ISTs e do HIV.

Os participantes do estudo PrEP Brasil percebem como alto o seu risco de infecção pelo HIV. Por isso, a pesquisadora discute a necessidade de vigilância epidemiológica no que diz respeito a monitorar a compensação de risco nos contextos reais de assistência à saúde. Ao longo de seu trabalho, Villela LM (2018) perpassa por estudos de ensaios clínicos randomizados importantes, que são referência sobre a PrEP.

Zucchi EM, et al. (2018), refletem sobre o perfil dos usuários da PrEP, suas vantagens em relação a outros métodos de barreira e os desafios para o uso contínuo, protetivo e seguro, a partir de resultados obtidos em estudos brasileiros e internacionais. Os autores analisaram alguns princípios vigentes nos estudos, como singularidade no cuidado, autonomia na escolha do método preventivo por parte do usuário, gestão do risco sexual, hierarquização de métodos preventivos e o papel da equipe multiprofissional para clarificar e embasar as escolhas dos usuários.

Os pesquisadores identificaram disparidades entre o que é orientado pelas diretrizes do Ministério da Saúde e o que de fato foi encontrado na estrutura dos serviços que permitem o acesso à profilaxia. A PrEP é um método serviço-dependente, e seu êxito depende da capacidade dos serviços de adotar uma organização do trabalho centrada nas necessidades dos usuários, tanto objetivas quanto subjetivas (ZUCCHI EM, et al., 2018).

Silva RATML (2018), em seu artigo, elabora uma análise sobre a necessidade da contribuição de movimentos sociais e da Defensoria Pública do estado de São Paulo no processo de incorporação da PrEP ao SUS. O pesquisador define o direito à PrEP como atinente ao direito à liberdade sexual, e elucida que a construção de um discurso de defesa da PrEP fortalece a luta por tal direito, bem como, de certa forma, atualiza os movimentos sociais de defesa dos direitos das Pessoas que Vivem com HIV/aids (PVHA) no cenário atual das políticas de saúde.

A tese de Costa SLM (2019) examinou a cobertura de dois jornais de grande repercussão nacional: O Globo (OG) e Folha de S. Paulo (FSP). O estudo resgatou conteúdos publicados sobre os métodos preventivos ao HIV: PrEP, Profilaxia Pós-Exposição (PEP) e o Tratamento como Prevenção (TcP), desde as primeiras notícias a respeito do tema até o fim de 2017.

O trabalho identificou a escassez de publicações jornalísticas acerca dos métodos preventivos, indicando que a cobertura jornalística esteve mais interessada nas descobertas científicas e nas ações dos órgãos internacionais e estatais ligados à aids e nos interesses da indústria farmacêutica em comercializar tais métodos, do que nas ações, críticas e anseios dos movimentos sociais, dos profissionais do serviço público de saúde e do público em geral (COSTA SLM, 2019).

Costa SLM (2019) dialoga sobre biomedicalização e biopoder, passando por noções como medicina alternativa, medicação, vulnerabilidade, farmacologização e saúde. É nesse sentido que se tem afirmado que a sociedade esteja vivendo a era da biomedicalização da aids, pois há um predomínio da visão tecnicista e biomédica acerca da epidemia, e, portanto, de suas soluções, marcada pela lógica do risco e da responsabilização individual pela saúde. 
A autora conclui que a PrEP foi o método preventivo com mais textos na FSP (12 a mais do que n'OG), além de ser, também, o método cujos textos ocuparam os maiores espaços em ambos, como de meia página a uma página inteira. Ao final de sua pesquisa, a autora avalia que estamos vivendo o recrudescimento do discurso moral sobre a aids como problema de saúde pública (COSTA SLM, 2019).

Carvalho CA e Azevêdo JHPC (2019), desenvolveram um levantamento das coberturas jornalísticas sobre movimentos das Lésbicas, Gays, Bissexuais, Transexuais, Queer, Intersexuais, Assexuais e Outras Identidades (LGBTQIA+), homofobia, HIV e aids. Os autores apresentam o histórico dos movimentos LGBTQIA+ brasileiros, indicando como a homofobia parece ser recorrente quando a aids está associada à essa população, particularmente, os homossexuais masculinos, as travestis e as transexuais.

O estudo aponta que os altos custos dos medicamentos constituem outra ordem de desafios, pairando sempre a incerteza da garantia de tratamento gratuito e universal, conquista de poucos países no mundo. Os pesquisadores destacam que, para alguns veículos de mídia, a PrEP vem sendo exposta como um abandono do sexo seguro, por meio de descrições moralistas e estereotipadas, o que constitui um desserviço, uma vez que fazem divulgação de informações errôneas que abrem precedentes para preconceitos e afetam diretamente uma questão pública de saúde (CARVALHO CA e AZEVÊDO JHPC, 2019).

Lermen HS, et al. (2019) realizaram uma análise de conteúdo descritivo e documental das campanhas nacionais de primeiro de dezembro (dia mundial da luta contra a aids) no período que compreende os anos de 2013 a 2017, visando identificar as representações da sexualidade e as formas de prevenção. No total, foram examinados 19 cartazes produzidos pelo Departamento de Doenças de Condições Crônicas e Infecções Sexualmente Transmissíveis (DCCl).

As mensagens nos materiais analisados colocam o sujeito como responsável por vigiar sua sexualidade, indicando a testagem como fator principal da prevenção ao HIV. Nos métodos de prevenção indicados nos cartazes, é notório uma diminuição da perspectiva do compromisso social e a maior responsabilização do sujeito (LERMEN HS, et al., 2019).

Os pesquisadores concluem que os aspectos relacionados ao uso do preservativo masculino não se mostram tão relevantes, pois, a lógica do "testar para se conhecer" individualiza o cuidado relacionado à prevenção. Verifica-se também uma oferta preventiva baseada no combo preservativo, testagem e as novas tecnologias como PrEP e PEP. Tal fato indica que a PC passou a orientar as campanhas do DCCI, contrariando as campanhas anteriores, que privilegiavam a populações-alvo e o uso prioritário da camisinha (LERMEN HS, et al., 2019).

Barreto VHS (2020), compartilha, em um artigo, os resultados de sua pesquisa de pós-doutoramento, em que acompanhou grupos virtuais e encontros presenciais de pessoas adeptas a práticas sexuais classificadas como "de risco". Dentro desse contexto, o pesquisador buscou identificar e discutir a respeito dos termos "responsabilidade", "consentimento" e "cuidado". A PrEP é retratada, no artigo, integrando a perspectiva da responsabilidade e do autocuidado de seus usuários.

Para o autor, ser saudável, é ter autocuidado e se proteger, a partir de uma perspectiva onde a responsabilidade sobre si e sobre as consequências de seus atos esteja presente. Ser saudável é poder ter escolhas livres, onde as opções de cuidados são dadas. A saúde está relacionada ao prazer que essa liberdade permite (BARRETO VHS, 2020).

No estudo "O reaparecimento da homossexualidade masculina nas estratégias de prevenção da infecção por HIV: reflexões sobre a implementação da PrEP no Brasil”, Barp LFG e Mitjavila MR (2020), utilizam-se do texto "O desaparecimento da homossexualidade" de Nestor Perlongher para estabelecer uma relação entre a PrEP e o "reaparecimento" da homossexualidade no Brasil.

A partir do surgimento da aids, a identidade homossexual masculina foi condenada. Os homossexuais vinham ao longo do tempo alcançando avanços em relação aos seus direitos (civis e humanos) e foram impactados negativamente a partir da associação da aids à uma doença considerara como "doença de gay". O surgimento de uma doença que afeta diretamente o desejo e práticas sexuais gerou um novo panorama acerca da homossexualidade nos consultórios médicos (BARP LFG e MITJAVILA MR, 2020). 
Os autores tomam como referência empírica o Protocolo Clínico de Diretrizes Terapêuticas para PrEP e relacionam com a perspectiva de noção de risco, onde para os autores, paradoxalmente, se reconhecer como potencialmente perigoso seria a única forma de se afastar de tal condição, permitindo então que a medicina discipline o corpo em suas práticas sexuais para que possa então gozar sem medo ou culpa (BARP LFG e MITJAVILA MR, 2020).

O estudo conclui que a PrEP possibilita o retorno dessa homossexualidade aos consultórios médicos, para serem vistos enquanto cidadãos e, mais do que isso, opera como objeto discursivo de problematizações de caráter sanitário e social (BARP LFG e MITJAVILA MR, 2020).

\section{CONSIDERAÇÕES FINAIS}

Os resultados obtidos no presente estudo, indicam a escassez de estudos atuais sobre a PrEP, que considerem aspectos particulares de contextos regionais das populações não metropolitanas. Para garantir os princípios fundamentais do SUS, as profilaxias precisam se articular com base nas particularidades das regiões não metropolitanas e em suas dimensões socioculturais. É necessário analisar continuamente a epidemia de HIV nos diferentes contextos brasileiros, com o intuito de entender quem são os potenciais e reais usuários da PrEP, suas demandas, para assim instrumentalizar práticas efetivas para o controle da epidemia e garantir o atendimento humanizado, inclusivo, acessível e integral para a população.

\section{REFERÊNCIAS}

1. BARP LFG, MITJAVILA MR. O reaparecimento da homossexualidade masculina nas estratégias de prevenção da infecção por HIV: reflexões sobre a implementação da PrEP no Brasil. Revista de Saúde Coletiva Physis, $2020 ; 30$.

2. BARRETO VHS. Responsabilidade, consentimento e cuidado. Ética e moral nos limites da sexualidade. Revista Sexualidad, Salud y Sociedad, 2020(35): 194-217.

3. CARVALHO CA, AZEVÊDO JHPC. Do AZT à PrEP e à PEP: aids, HIV, movimento LGBTI e jornalismo. Revista Eletrônica de Comunicação, Informação e Inovação em Saúde, 2019; 13(2).

4. COSTA SLM. Risco, biomedicalização e aids: cobertura jornalística sobre métodos biomédicos de prevenção ao HIV, Tese (Doutorado em Informação, comunicação e saúde). Instituto de Comunicação e Informação Científica e Tecnológića em Saúde, Rio de Janeiro, 2019; 281 p.

5. COSTA-JÚNIOR FM. Geração, masculinidades e atenção primária à saúde em três cidades do Nordeste brasileiro, Tese (Doutorado em Ciências). Universidade de São Paulo, São Paulo, 2014; 238 p.

6. FACCHINI R, PINHEIRO TF, CALAZANS GJ. Prevenção de HIV/Aids, produção de diferenças e processos de mudança social. Sexualidad, Salud y Sociedad, 2018; 29: 253-62.

7. KNAUTH DR, et al. O diagnóstico do HIV/aids em homens heterossexuais: a surpresa permanece mesmo após mais de 30 anos de epidemia. Cadernos de Saúde Pública, 2020; 36: e00170118.

8. LERMEN HS, et al. Aids em cartazes: representações sobre sexualidade e prevencão da aids nas campanhas de $1^{\circ}$ de dezembro no Brasil (2013-2017). Interface-Comunicação, Saúde, Educação, 2019; 24: e180626.

9. LOPES JDS, et al. Pré-exposição (prep) ao hiv e indivíduos em maior vulnerabilidade: uma revisão crítica da literatura de 2013 a 2018. Revista Eletrônica Acervo Saúde, 2019; 27: e963.

10. MAKSUD I, et al. Tecnologias de prevenção do HIV e desafios para os serviços de saúde. Revista Brasileira de Epidemiologia, 2015; 18: 104-119.

11. MINISTÉRIO DA SAÚDE. Secretaria de Vigilância em Saúde. Boletim Epidemiológico HIV/Aids. 2020. Disponível em: http://www.aids.gov.br/pt-br/pub/2019/boletim-epidemiologico-de-hivaids-2019. Acessado em 10 de abril de 2020.

12. MONKEN M, et al. O território na saúde: construindo referências para análises em saúde e ambiente. In: MIRANDA AC, et al. Território, ambiente e saúde. 1 a ed. Rio de Janeiro: Editora Fiocruz, 2008; 23-41 p.

13. PARKER RG. Na contramão da aids: sexualidade, intervenção, política. São Paulo: Editora 34; 2000.

14. POLIDORO M, et al. O panorama atual da estratégia da profilaxia pré-exposição (PrEP) no Brasil e os caminhos possíveis para busca da equidade em saúde. Saúde \& Transformação Social/Health \& Social Change, 2020; 11(1): 001-011.

15. QUEIROZ AAFLN, et al. Conhecimento sobre HIV/Aids e implicações no estabelecimento de parcerias entre usuários do Hornet巴. Revista Brasileira de Enfermagem, 2018; 71(4): 2062-70.

16. SANTOS M. A natureza do espaço: técnica e tempo, razão e emoção. São Paulo: Hucitec, 1999.

17. SILVA RATML. Diversidade e liberdade sexual: Defensoria Pública, movimentos sociais e a PrEP no SUS. Serviço Social \& Sociedade, 2018; 132: 346-61.

18. SOUZA MAA. Uso do território e saúde: refletindo sobre "municípios saudáveis". In: SPERANDIO AMG. O processo de construção da rede de municípios potencialmente saudáveis. 1a ed. Campinas: IPES, 2004; 57-77p.

19. VILLELA LM. Percepções sobre o uso da profilaxia pré-exposição ao HIV (PrEP) e possível compensação de risco entre homens que fazem sexo com homens (HSH) e mulheres transexuais (TRANS) potencialmente elegíveis para o uso de PrEP no estudo PrEP Brasil. Dissertação (Mestrado em Ciências) - Instituto Nacional de Infectologia Evandro Chagas - Fundação Oswaldo Cruz, Rio de Janeiro, 2018; 60 p.

20. ZUCCHI EM, et al. Da evidência à ação: desafios do Sistema Único de Saúde para ofertar a profilaxia pré-exposição sexual (PrEP) ao HIV às pessoas em maior vulnerabilidade. Cadernos de Saúde Pública, 2018. 